\title{
La participación política de las mujeres migrantes a través de las asociaciones en Galicia. Liderazgo y relaciones de poder
}

\section{Luzia Oca González}

Centro de Estudos Transdisciplinares para o Desenvolvimento (CETRAD)* luziag@utad.pt

\author{
Xabier Lombardero \\ Universidade da Coruña \\ xabilombardero@gmail.com
}

\author{
Recepción: 16-10-2017 \\ Aceptación: 02-03-2018
}

Publicación: 28-06-2018

\section{Resumen}

En este artículo se analizan las relaciones de género en las asociaciones de migrantes en Galicia en tanto que principales formas organizativas de la participación social y política de esta población. Partiendo de una tipología de las asociaciones que considera en primer término su carácter mixto o no, así como el liderazgo femenino o su ausencia, se estudiarán tanto las relaciones generizadas de poder que sustentan esta tipología como otros elementos que explican la participación política de las mujeres migrantes: la división sexual del trabajo entre las personas migrantes, los sistemas de sexo y género, la experiencia asociativa en origen y las políticas públicas en materia de integración, especialmente las del ámbito local. Los resultados de este análisis apuntan a que el papel preeminente de las mujeres en las asociaciones mixtas no obedece a una concienciación emancipadora, sino más bien a factores circunstanciales, mientras que el surgimiento de otras exclusivamente femeninas responde a una fuga del machismo en sus comunidades. La metodología de esta investigación combina, de manera secuencial, material empírico de corte cuantitativo (censo de asociaciones de migrantes y cuestionarios telefónicos) y cualitativo (entrevistas semidirigidas a líderes asociativos — hombres y mujeres), así como un estudio de caso etnográfico.

Palabras clave: género; asociacionismo migrante; relaciones de poder; liderazgo; participación política; instrumentalización

* Este trabajo ha sido financiado por los Fondos Europeos Estructurales y de Inversión, en su componente FEDER, a través del Programa Operacional Competitividad e Internacionalización (COMPETE 2020) (Proyecto n. ${ }^{\circ} 006971$ [UID/SOC/04011], referencia de financiación: POCI-01-0145-FEDER-006971), y por Fondos Nacionales a través de la FCT (Fundación para la Ciencia y la Tecnología), en el ámbito del Proyecto UID/ SOC/04011/2013. 


\begin{abstract}
Political participation of migrant women through associations in Galicia: Leadership and power relations
\end{abstract}

This article analyzes gender relations in migrant associations of Galicia as the main organizational forms of social and political participation of this population. Starting from a typology of associations that considers, first and foremost, their mixed nature or not, as well as the presence or absence of female leadership, we study both the gendered power relations that underpin this typology and other elements that explain the political participation of migrant women: the sexual division of labor among migrants, sex-gender systems, previous associative experience at origin and public policies on integration, especially at the local level. The results of the analysis suggest that the preeminent role of women in mixed associations is not due to emancipatory awareness but rather to circumstantial factors, while the emergence of other exclusively female ones responds to an escape from the machismo in their communities. The research methodology sequentially combines quantitative (census of migrant associations and telephone surveys) and qualitative (semi-directed interviews with male and female associative leaders) empirical material, as well as an ethnographic case study.

Keywords: gender; migrant associationalism; power relationships; leadership; political participation; instrumentalization

\title{
Sumario
}

1. Aproximación a la cuestión del asociacionismo migrante desde el contexto gallego y español con enfoque de género

2. Una tipología de las asociaciones de migrantes en Galicia desde la perspectiva de género
3. Estudio de caso etnográfico: mujeres, asociacionismo migrante y políticas locales en Burela

4. Conclusiones y futuras líneas de investigación

Referencias bibliográfica

El presente trabajo tiene como objeto la presencia y la participación de mujeres en las asociaciones migrantes ${ }^{1}$ en Galicia. Se basa en una serie de datos obtenidos en dos procesos de investigación diferenciados. En primer lugar, materiales empíricos reunidos para el proyecto de investigación Asociacionismo migrante en Galicia: Lazos transnacionales y codesarrollo desde una perspectiva de género. Concretamente, se han utilizado los resultados del censo de asociaciones de migrantes, así como el cuestionario telefónico realizado a treinta entidades asociativas y ocho entrevistas semidirigidas a presidentas de asociaciones ${ }^{2}$. Por otra parte, se incorporan los resultados de una investigación etnográfica realizada desde 1998 en la localidad de Burela (Lugo) en torno a sus comunidades

1. Se emplea este término, en lugar de inmigrante, de forma consciente para incluir a personas retornadas, que, en el contexto gallego, tienen una fuerte presencia en los flujos de movilidad humana.

2. Este artículo forma parte de un monográfico sobre participación política de las comunidades migrantes en España. Las cuestiones metodológicas se comentan al detalle en un capítulo inicial del mismo, por lo que no profundizaremos mucho en este aspecto en el presente texto. 
migrantes, que ha incluido la participación en diversos proyectos y actividades, así como una intensa observación participante.

Una vez contextualizada la realidad de la configuración de las asociaciones de migrantes en Galicia, nos centraremos en elaborar una tipología teniendo en cuenta su composición y su representación en términos de género, a partir de la cual analizaremos los elementos más relevantes en la participación de las mujeres y sus relaciones de poder dentro de las mismas con respecto a los hombres. Seguidamente, nos centraremos en un caso etnográfico, que, además de buscar explicaciones a la preeminencia de organizaciones migrantes regidas por mujeres, retrata la estrecha relación entre asociacionismo migrante y poder local, a través principalmente de las mujeres, en una suerte de instrumentalización política de estas organizaciones.

\section{Aproximación a la cuestión del asociacionismo migrante desde el contexto gallego y español con enfoque de género}

La incorporación de las personas migrantes a las sociedades de acogida, en este caso concretamente a la gallega y en general a la española, es un proceso que abarca diferentes fases que estan marcadas por intereses y necesidades cambiantes, tanto de las propias personas como de los distintos agentes sociales con los que se encontrarán durante el trayecto migratorio y en el contexto de llegada (Martínez de Lizarrondo, 2009). Es decir, las condiciones que rodean la llegada de las personas migrantes evolucionan con el tiempo, tanto en los aspectos legales, laborales o personales como en el propio trayecto migratorio, pues no es lo mismo hacerlo de manera regular o no.

En esta investigación nos centraremos más en la fase que tiene que ver con los procesos de inserción social, una vez el trayecto migratorio se consolida con el asentamiento en el país de destino, en donde desempeñan un papel fundamental las asociaciones de migrantes en tanto que principales formas organizativas de la participación social y política de esta población en el contexto de acogida. Por ello, nos centraremos en la configuración de dichas asociaciones, en su papel dentro de la propia comunidad migrante y en las relaciones que se dan en ellas desde una perspectiva de género y feminista.

Conviene profundizar en una cuestión que muchas veces ronda ciertos discursos y prácticas en las políticas vinculadas a la cuestión migratoria. El intento de usar la medida de las respectivas tasas de asociacionismo para comparar los niveles de integración social y política de distintos sectores de una población, como pretendieron algunos estudios (Putman, 1993), no conduce a resultados válidos, si entre esos sectores no existe una similitud o cierto grado de coincidencia en cuanto a lo que para ellos significan las propias asociaciones. Es decir, para una persona europea residente en España, por ejemplo, el participar en una asociación de su país de origen no significará lo mismo que para una persona inmigrante marroquí.

Así pues, al contrario que en las perspectivas planteadas por Putman, no observamos en las tasas de asociacionismo una medida de integración social, 
más bien interpretamos que las situaciones sociales de los diversos colectivos son las que pueden movilizar sus pertenencias asociativas. Al analizar las asociaciones gallegas en el siguiente apartado, se constatan las tesis de Aparicio y Tornos (2010: 60-61) que demostraron que cuanto más extraña es a la sociedad de origen la condición identitaria de un colectivo, más asociaciones crea ese colectivo. Por otro lado, cuanto más comunitarista haya sido la socialización de los miembros de un colectivo, más asociaciones creará al llegar a destino, mientras que cuanto más individualista sea, menos creará.

Previamente, conviene señalar que las decisiones políticas en materia migratoria han marcado la evolución del fenómeno de movilidad internacional e influyen sobre el nivel de integración de la población migrante. La aceleración de las llegadas, las regularizaciones y las características de la composición de la población extranjera no son procesos que se den sin más, por cuestiones de azar, sino que están relacionados con las necesidades laborales y las preferencias políticas. Además, en el caso del Estado español, las políticas migratorias siempre han estado orientadas por las necesidades del mercado de trabajo, puesto que la economía española siempre ha mostrado una alta dependencia hacia mercados irregulares. De ahí que, en su momento, se facilitase el asentamiento de inmigrantes irregulares por la necesidad de cubrir cuotas de empleo fuera de la legalidad en determinados sectores como la construcción y la hostelería o, en los últimos tiempos, con el trabajo de cuidados a través de las empleadas del hogar (Martínez Buján, 2008).

En este aspecto, resulta importante destacar que las mujeres, en cuanto sujetos políticos, carecen en la práctica de una posición de igualdad real. Las barreras existentes en la accesibilidad a los espacios de toma de decisiones y a los cargos de elección popular están directamente relacionadas con la división sexual del trabajo en los espacios públicos y privados. En la sociedad gallega, así como en la española, es patente la desigualdad de género en las distintas esferas de la vida privada y pública. En el caso de las mujeres inmigrantes estas barreras también vienen marcadas por los sistemas de sexo y género de sus sociedades de origen. En el campo migratorio, las mujeres tienen presencia y participan en asociaciones de forma voluntaria, incluso en cargos de responsabilidad, cumpliendo en muchas ocasiones un papel que no les es nuevo. Pero no son destinatarias como ciudadanas de pleno derecho y se «naturaliza» en gran medida como extensión del trabajo de cuidados, asignado históricamente a las mujeres. Así pues, el problema de los cambios en el papel público y en el liderazgo femenino alude y convoca a profundos cambios en el conjunto de la sociedad, lo que, en último caso, significa cambios en la identidad y en las condiciones de género de los hombres a nivel global.

Este trabajo pretende centrarse en las asociaciones de migrantes, pues sabido es que la población migrante, una vez instalada, tiende a crear grupos y asociaciones más o menos formales, organizadas generalmente según el origen nacional o étnico, para dar respuesta a necesidades y a anhelos diversos. En este sentido, las asociaciones se han constituido en un elemento clave en la articulación de la vida cotidiana del colectivo inmigrante actuando a dos niveles. Por 
un lado, sirven como herramienta para la relación con la sociedad de acogida y sus instituciones, es decir, funcionan como una instancia de representación e intermediación con las administraciones públicas, pero también con la propia sociedad civil del país de destino. Por otro lado, hacia dentro del colectivo, suelen servir para redefinir, a veces de manera conflictiva, las relaciones internas dentro del nuevo contexto de asentamiento. Partimos de la idea de que la identidad es un proceso de construcción que se ve afectado, en este caso, por el contexto de acogida, lo que permite reorganizar y redefinir cuestiones que no tendrían cabida si permaneciesen en sus sociedades de origen (Gadea y Albert, 2011).

Las políticas de integración son competencia de las administraciones autonómicas, y la capacidad externa de influencia desde las organizaciones sociales es escasa. En el contexto español, la estructuración de los procesos de coordinación y evaluación de los planes de integración de inmigrantes reflejan notables diferencias entre lo planteado y lo realizado. La mayoría de las veces las asociaciones de migrantes son instrumentalizadas por dichas políticas y los agentes institucionales que las aplican (Martínez de Lizarrondo, 2008), hecho que pudimos constatar a lo largo de la presente investigación y que explicaremos con mayor detalle en el estudio de caso.

En el caso gallego, las competencias en materia de política para la población inmigrante han variado de departamento desde el año 2000. En la actualidad se adscriben a la Consellería de Política Social, a través de la Dirección Xeral de Familia e Inclusión, dentro de la que existe un servicio de inmigración que cuenta con una escasa actividad, más allá de subvencionar la actividad de algunos municipios a través de convenios. Las intervenciones con colectivos migrantes han sido desarrolladas mayoritariamente desde las administraciones locales y desde algunas organizaciones no gubernamentales, a través de fondos autonómicos, estatales o comunitarios. No existen estructuras de participación en las que las organizaciones migrantes puedan tener voz en asuntos de política migratoria. En 2003 nació el Foro Galego de Inmigración, asamblea abierta constituida por más de 80 asociaciones inmigrantes y entidades sociales, sindicales y políticas, organizadas colectivamente para defender los derechos de las personas inmigrantes, sirviendo como lugar de encuentro y debate de los distintos colectivos. Sus recomendaciones no son vinculantes con la política autonómica.

Por otro lado, la Secretaría Xeral de Igualdade de la Xunta, organismo que concentra las competencias en materia de igualdad de género, no cuenta con ninguna línea de trabajo específica con mujeres inmigrantes. En el año 2017 ninguna asociación de mujeres inmigrantes fue contemplada en la concesión de ayudas a asociaciones de mujeres en Galicia. Tampoco en la convocatoria a entidades para financiar programas dirigidos a mujeres en situación de especial vulnerabilidad, grupo en el que la Administración encuadra a las migrantes.

Llegadas a este punto, conviene destacar una característica propia del contexto territorial en el que se desarrolla la investigación, como es que, a lo largo de su historia, Galicia siempre ha presentado una gran influencia de 
movimientos migratorios, sobre todo los relacionados con flujos de salida. La emigración constituía una «industria de los pobres» que estaba perfectamente integrada en el ethos cultural de las zonas campesinas del norte de la Península (Soutelo, 2011). O, por dejarlo más claro, Galicia siempre exportó carne de vaca y carne humana (Cardesín, 1992), por lo que, en la actualidad, más allá de los flujos de entrada que han afectado a todo el Estado y que han remitido a partir de la crisis económica de 2008, en Galicia se mantiene una especial incidencia en cuanto a movimientos migratorios vinculados al retorno, tanto de personas que habían salido como de sus familiares y descendientes, lo que se ve claramente reflejado en el panorama asociativo.

En el contexto gallego, las corrientes de retorno han crecido proporcionalmente a medida que la mejora de los medios de transporte ha ido reduciendo el esfuerzo, material y temporal, que supone para las personas migrantes viajar entre los lugares de destino y de origen, principalmente vinculados a Latinoamérica. Además, dicho proceso se ha visto afectado por la coyuntura que se está viviendo en determinados países, como Venezuela o Brasil, lo que provoca que descendientes de gallegos decidan regresar.

\section{Una tipología de las asociaciones de migrantes en Galicia desde la perspectiva de género}

Partiendo de los datos recogidos durante el trabajo de campo realizado en 2014, elaboraremos una tipología de las asociaciones activas en Galicia en ese momento, a partir de un cuestionario telefónico realizado a 30 de ellas. Estas forman parte de un censo más amplio de organizaciones de migrantes gracias al que sabemos que había 79 asociaciones activas en Galicia en el momento de la investigación.

Existen muy pocos estudios previos que analicen la configuración de estas asociaciones desde una perspectiva de género, lo cual ha despertado nuestro interés y nos ha llevado a analizar la estructura y la organización interna a partir de su composición y representación. La tipología elaborada considera en primer término su carácter mixto o no, teniendo en cuenta el sexo de las personas asociadas. En segundo lugar, se tendrá en cuenta la presencia o ausencia de liderazgo femenino y la representatividad de las mujeres en las juntas directivas.

Los datos del cuestionario fueron complementados con los obtenidos a través de las entrevistas a ocho mujeres que ostentan la presidencia de algunas de estas asociaciones. A partir de ellos, se estudiarán tanto sus motivaciones como otros elementos que puedan servir de factor explicativo a la hora de abordar la participación política de las mujeres migrantes en el contexto asociativo gallego actual.

En cuanto a la composición de las 30 asociaciones inquiridas, la gran mayoría (25) son mixtas, formadas por hombres y mujeres, mientras que tres son estrictamente femeninas y dos masculinas.

De acuerdo con las entrevistas realizadas a sus dirigentes, tanto la asociación de mujeres de origen marroquí Al Amal (Marín) como la de origen senegalés 
Cuadro 1. Composición de las asociaciones por sexos

\begin{tabular}{lr}
\hline Mixtas & 25 \\
Femeninas & 3 \\
Masculinas & 2 \\
Total & 30 \\
\hline
\end{tabular}

Fuente: elaboración propia a partir del cuestionario a las entidades asociativas.

Domu Africa (A Coruña) surgieron como respuesta a la imposibilidad de trabajar dentro de las asociaciones mixtas de su nacionalidad, dominadas habitualmente por hombres y muy centradas en prácticas religiosas. A pesar de ello, en ambas asociaciones reconocen que cuentan con presencia y con colaboraciones masculinas puntuales y de forma muy minoritaria.

Además, a partir de la información obtenida con las entrevistas, se percibe una cierta resignación (o impotencia) ante el poder de los hombres, que se supera a través de la propia cooperación entre las mujeres, lo que lleva a la creación de asociaciones integradas exclusivamente por ellas, y/o a reivindicaciones embrionarias, al menos en el plano discursivo (Molina et al., 2013: 156). Esto evidencia las dificultades para superar los esquemas de segregación sexual de las sociedades de origen, en este caso determinadas por el factor religioso, de manera que la única hipótesis de una cierta emancipación o autonomía se da a través de la creación de asociaciones de mujeres, con lo que se constata cómo ellas aprovechan las condiciones que presenta la sociedad de acogida, con la negativa de las senegalesas a seguir los mandatos de género imperantes en su país, para modificar los roles que arrastran y son típicos en su sociedad de origen.

Primero había una asociación que se llamaba África Universal, y cuando las chicas fueron, yo no fui. Las chicas fueron y no las querían meter en la junta directiva y tal, ellas protestaban y decían: "¿Por qué no, por qué no sé qué?». Yo les dije: "Pues tenéis razón. Ahora estamos en España, no dejáis que os manden, tenéis que tal...». Y luego, a la hora de nombrar los puestos, yo estaba allí de casualidad. (Entrevista a la presidenta Domu África)

En la creación de la asociación de mujeres marroquíes Al Amal de Marín (Pontevedra) existe una cierta influencia de la trabajadora social municipal y de la oportunidad y necesidad de constituirse formalmente para poder optar a recursos sociales, como local o subvenciones. También para representar su cultura en actividades organizadas por la institución local, en un municipio en el que la migración está muy ligada al sector pesquero. Se constata así que la consolidación de estas organizaciones muchas veces está condicionada por el entorno institucional y social, así como por dinámicas internas de la propia comunidad y del contexto social, y que experimentan constantes procesos de reestructuración, bien sean de fisión o de fusión (Garreta, 1998), como veremos en el estudio de caso de la localidad de Burela, de donde es la tercera de 
Cuadro 2. Composición de las juntas directivas por sexos

\begin{tabular}{lr}
\hline Solo mujeres & 7 \\
Solo hombres & 7 \\
Paritaria & 2 \\
Mixtas con más de un 50\% de hombres & 7 \\
Mixtas con más de un 50\% de mujeres & 7 \\
Total & 30 \\
\hline
\end{tabular}

Fuente: elaboración propia a partir del cuestionario a las entidades asociativas.

estas asociaciones de mujeres, formada por caboverdianas e integrada por las diez componentes de un grupo de música tradicional.

En los dos primeros casos comentados de asociaciones estrictamente femeninas, se verifica la hipótesis de trabajo de Molina et al. (2013), que, en una investigación sobre liderazgo en asociaciones de migrantes de origen africano, encontraron una clara diferencia de objetivos entre las asociaciones masculinas y las femeninas, que atribuyen al impacto de la desigualdad entre las posiciones sociales de hombres y mujeres, debido a las características de las propias culturas de género en las sociedades de origen, en este caso Senegal y Marruecos. Por tanto, este asociacionismo africano es joven y no responde a una única forma organizativa, sino que, en función de dinámicas internas y externas, intereses de los asociados y años de vida de cada asociación, adquiere diferentes estructuras y estrategias (Garreta y Llevot, 2013).

Una vez analizada la base asociativa, si se presta atención al sexo de las personas representadas en los órganos de poder de estas 30 asociaciones, vemos que se repite la misma cifra (7) para las que se encuentran representadas exclusivamente por mujeres o por hombres. La misma cifra se repite nuevamente en el caso de las juntas directivas en las que las mujeres sobrepasan o no alcanzan el 50\% de representatividad. En cuanto a las estructuras representativas paritarias, tan solo existen en dos de las asociaciones, como se puede comprobar en el siguiente cuadro.

Los datos recogidos en los cuestionarios parecen indicar un cierto equilibrio de género en la representación asociativa, en la que llama la atención la coincidencia en las cifras, aunque quizás lo que habría que destacar sea la aparente segregación y el escaso peso de las estructuras paritarias, en las que hombres y mujeres comparten responsabilidades de forma equitativa.

En cuanto a las siete asociaciones cuya junta directiva es netamente femenina, tres de ellas están formadas exclusivamente por mujeres migrantes, de las que hemos hablado anteriormente. Las restantes presentan una composición mixta y entre ellas se encuentra una asociación venezolana de Vigo y tres de Burela (una caboverdiana y dos peruanas).

Al cruzar los datos del cuestionario y las entrevistas, llama la atención la existencia de $\operatorname{cinco}^{3}$ asociaciones migrantes en esta localidad de la costa de

3. Una de las asociaciones caboverdianas no forma parte de la muestra que respondió el cuestionario, aunque han sido entrevistadas sus dirigentes. 
Cuadro 3. Composición, origen y localidad de las asociaciones regidas por mujeres

\begin{tabular}{lll}
\hline Composición & Origen & Localidad \\
\hline Femenina & Senegal & A Coruña \\
Femenina & Marruecos & Marín (Pontevedra) \\
Femenina & Cabo Verde & Burela (Lugo) \\
Mixta & Cabo Verde & Burela (Lugo) \\
Mixta & Perú & Burela (Lugo) \\
Mixta & Perú & Burela (Lugo) \\
Mixta & Venezuela & Vilagarcía (Pontevedra) \\
\hline
\end{tabular}

Fuente: elaboración propia a partir del cuestionario a las entidades asociativas.

Lugo, con un peso muy relevante de mujeres. Tres de ellas están integradas por migrantes de origen caboverdiano y dos por personas peruanas. Todas ellas son mixtas, excepto una de las caboverdianas. En todas ellas las juntas directivas están formadas por mujeres ${ }^{4}$. Posteriormente nos detendremos en este caso excepcional, intentando comprender los motivos que han llevado a que las mujeres caboverdianas y peruanas se convirtiesen en las representantes de sus organizaciones comunitarias.

En cuanto a los objetivos y a las actividades que realizan las siete asociaciones regidas por miembros del colectivo femenino, ninguna de ellas presenta una perspectiva de trabajo específicamente orientada a la igualdad de género, excepto la marroquí, que realiza acompañamiento a mujeres de forma integral. Todas las demás señalan estar más centradas en la integración, sobre todo en el ámbito escolar de sus descendientes y en el trabajo con familias. También en asesoramiento sobre cuestiones legales y administrativas y formación laboral (venezolana y senegalesa). No menos importantes son las cuestiones que posibilitan mantener y difundir prácticas culturales de sus países de origen. Recordemos que una de estas organizaciones es en realidad un grupo de música tradicional.

Por tanto, la existencia de directivas estrictamente femeninas no se corresponde con un objetivo de lucha a favor de los derechos de las mujeres o de la mejora de sus situaciones, sino que tiene que ver con las capacidades de las mujeres que las encabezan o con aspectos coyunturales. Así lo señala otro estudio (Molina et al., 2013) referido a Catalunya, Valencia, Navarra y La Rioja.

Continuando nuestro análisis de la representación asociativa, encontramos un grupo de siete asociaciones representadas exclusivamente por hombres, todas ellas de origen africano.

En cuanto a la composición de su base asociativa, dos de ellas no cuentan con mujeres entre sus asociados y en otras dos representan menos del 10\% de sus integrantes, mientras en las tres restantes suponen menos del $50 \%$.

4. Una de las asociaciones peruanas cuenta con dos hombres en su junta directiva, en sendas vocalías, pero los cuatro puestos representativos principales están ocupados por mujeres, por lo que es considerada como de liderazgo femenino. 
Cuadro 4. Composición, origen y localidad de las asociaciones regidas por hombres

\begin{tabular}{lll}
\hline Composición & Origen & Localidad \\
\hline Masculina & Argelia & Lalín (Pontevedra) \\
Masculina & Marruecos & Pontevedra \\
Mixta (-50\% mujeres) & Sáhara Occidental & Ourense \\
Mixta $(-50 \%$ mujeres) & Senegal & Vigo \\
Mixta $(-50 \%$ mujeres) & Senegal & A Coruña \\
Mixta (-10\% mujeres) & Senegal & A Coruña \\
Mixta (-10\% mujeres) & Senegal & A Coruña \\
\hline
\end{tabular}

Fuente: elaboración propia a partir del cuestionario a las entidades asociativas.

Por tanto, se trata de espacios asociativos sin apenas lugar para las mujeres, mucho menos fuera de sus roles tradicionales de género vinculado al trabajo de cuidados. Tres de estas organizaciones son magrebíes (Argelia, Marruecos y Sáhara) y otras cuatro del África subsahariana (Senegal). Su creación responde a unos objetivos similares que tienen que ver con la prestación de ayuda y asesoramiento administrativo entre iguales, tanto en relación con las autoridades españolas como con sus países de origen, a través de los consulados respectivos ${ }^{5}$.

Otro de los objetivos importantes es el refuerzo y el mantenimiento de lazos socioculturales propios, pues todas ellas son de nacionalidades donde la lengua y la religión son distintas al país de acogida, con lo que se confirma la hipótesis de Aparicio y Tornos (2010), según la cual cuanto más extraña es a la sociedad de origen la condición identitaria de un colectivo, más asociaciones crea. En estos casos existe un claro interés por no perder sus raíces ni sus idiomas, bien sea el árabe o el wolof, incluso el francés, aunque dos de ellas desarrollan actividades de alfabetización en castellano para sus miembros. Por otro lado, solo una de estas organizaciones, la argelina, tiene estrictamente carácter religioso, aunque en todo este grupo dicho elemento es fundamental.

En tercer lugar, recogidas en el cuadro 5, existen ocho asociaciones que presentan una mayoría de mujeres en su junta directiva. Es decir, la mitad o más de las personas que forman parte de su junta directiva son mujeres. De estas, cuatro son asociaciones de origen latino: dos peruanas, una venezolana y una uruguaya. Otras tres son de origen africano, todas senegalesas, y una europea, de Polonia. Las latinas y la polaca asientan su liderazgo mayoritariamente en mujeres debido a circunstancias coyunturales o bien por experiencias vitales previas de las propias mujeres, que ya ejercían un papel del liderazgo en sus países. En el caso de las senegalesas, responden más a la realidad segregada de su comunidad de origen y a la necesidad de integración de sus hijos en la cultura de destino, así como, de nuevo, a la conservación de los lazos culturales con su país de origen.

5. Algunas de estas asociaciones se constituyeron debido a la necesidad de repatriar cadáveres de los migrantes que morían en suelo gallego pero querían ser enterrados en su población natal. 
Cuadro 5. Origen y localidad de las asociaciones (mixtas) regidas mayoritariamente por mujeres

\begin{tabular}{ll}
\hline Origen & Localidad \\
\hline Mixto (latino, africano, europeo) & Ferrol \\
Polonia & Santiago \\
Uruguay & Ferrol \\
Uruguay & Carballo \\
Venezuela & A Estrada (Pontevedra) \\
Perú & A Coruña \\
Senegal & Lugo \\
\hline
\end{tabular}

Fuente: elaboración propia a partir del cuestionario a las entidades asociativas.

Cuadro 6. Origen y localidad de las asociaciones (mixtas) regidas mayoritariamente por hombres

\begin{tabular}{ll}
\hline Origen & Localidad \\
\hline Mixto (latino, africano) & A Coruña \\
Cuba & Pontevedra \\
Argentina & A Coruña \\
Venezuela & A Coruña \\
Venezuela & Ourense \\
República Dominicana & Viveiro (Lugo) \\
República Dominicana & Culleredo (A Coruña) \\
\hline
\end{tabular}

Fuente: elaboración propia a partir del cuestionario a las entidades asociativas.

En este aspecto conviene destacar una característica propia del contexto migrante gallego ya apuntada en el apartado inicial del presente artículo, como es el hecho del retorno en los flujos migratorios sobre todo con países de América Latina, como Uruguay o Venezuela, y que también veremos en el siguiente cuadro, referido a las asociaciones regidas mayoritariamente por hombres.

Finalmente, identificamos siete asociaciones con mayoría de hombres en sus órganos directivos; seis de ellas de origen latino y vinculadas a corrientes de retorno, excepto en el caso dominicano, y una mixta, en la que predominan personas de Guinea Ecuatorial, Ecuador, Cuba y Perú. Las actividades que desarrollan las de origen latino están muy vinculadas con aspectos folclóricos de su cultura de origen, como el mantenimiento de tradiciones y fiestas, así como aspectos legales y de incidencia en sus países de origen, como la participación en elecciones o el contacto permanente con el consulado o la embajada.

Así pues, una vez analizados los datos del cuestionario y cruzados estos con la información obtenida en las ocho entrevistas en profundidad realizadas, podríamos afirmar que la experiencia migratoria como hecho social total involucra diferentes niveles y planos de la realidad. Y, en el caso que nos ocupa, que es analizar la configuración de asociaciones de migrantes desde una perspectiva de género en Galicia, también se percibe de esta forma. Es decir, como 
se constata en otras investigaciones, la realidad de las personas migrantes y de las asociaciones que conforman suele estar sujeta a las modificaciones en los modelos económicos y culturales, tanto en el ámbito de las prácticas como en el de las representaciones sociales (Gadea y Albert, 2011). Sin embargo, estas prácticas y representaciones tienen diferentes repercusiones para el sujeto según su género, su etnia y su clase social de procedencia y de inserción.

Los datos cuantitativos analizados permiten realizar un análisis limitado en cuanto a su profundidad, a la hora de comprender cómo se desarrollan y se operan las relaciones de género en el interior de las organizaciones. Entre otros aspectos, no permiten entender debidamente la influencia de otros actores importantes en el devenir de las asociaciones migrantes, como pueden ser los institucionales, políticos y sociales. Por ello se presenta a continuación un caso de estudio, captado a través del método etnográfico, que pone de relieve los intereses que mueven la actuación de administraciones públicas en su relación con las asociaciones migrantes, así como las propias relaciones entre diferentes organizaciones en el espacio local y autonómico.

\section{Estudio de caso etnográfico: mujeres, asociacionismo migrante y políticas locales en Burela}

A continuación nos detendremos en el análisis de un caso concreto de asociacionismo migrante en Galicia, que supone una excepción en el panorama asociativo que acabamos de analizar. Se sitúa en Burela, localidad del centro de la costa de Lugo, conocida en la sociedad gallega como ejemplo de integración de los colectivos migrantes. En la localidad existe tradición asociativa desde finales de la década de 1980, cuando nació la primera organización migrante, formada por familias caboverdianas. En la actualidad, existen cinco asociaciones de este tipo, tres de Cabo Verde y dos peruanas, todas ellas encabezadas por mujeres.

El estudio que se presenta está basado, además de en los cuestionarios y en las entrevistas citados, en un largo trabajo de campo etnográfico iniciado en 1998 con la comunidad caboverdiana, que coincidió inicialmente con un proyecto de intervención. En el análisis tendremos en cuenta tres de las funciones que comúnmente se atribuyen las asociaciones migrantes: mediación política, identidad y solidaridad (Rex, 1994; Albert, 2004, Merino, 2005).

\subsection{Burela: La pesca, motor de la inmigración}

Burela experimentó un proceso de acelerado crecimiento demográfico, urbanístico y económico a partir de la década de 1960. Su puerto, uno de los más importantes de Galicia y del Cantábrico desde hace décadas, ha funcionado como verdadero foco de atracción de mano de obra inmigrante desde finales de los años setenta, lo que posibilitó el asentamiento de una comunidad de origen caboverdiano a partir de 1978. Otros subsectores vinculados a los servicios (hostelería), a los cuidados y en menor medida a la construcción han reforzado la demanda de mano de obra extranjera en las dos últimas décadas, 
caracterizadas por una diversificación de los flujos y un aumento exponencial de la población extranjera.

El municipio, que cuenta en la actualidad con una población cercana a los 10.000 habitantes (INE), presenta una de las tasas más elevadas de población inmigrante de Galicia, que solo recientemente ha experimentado una leve caída. Partiendo de un 2,7\% de población migrante en 2000, creció ininterrumpidamente durante la primera década del siglo XXI y alcanzó su punto álgido en 2013 con un 12,7\%, para quedarse en un 11,3\% en 2015, según los datos del Padrón (INE).

La evolución de la comunidad caboverdiana de Burela ha sido analizada desde la antropología aplicada (Oca, 2013), destacando el papel fundamental de las mujeres en la organización de sus grupos domésticos y las relaciones intracomunitarias y externas al colectivo. La razón de esta preeminencia de las mujeres se ha explicado por la inserción laboral de sus maridos en la pesca de altura, único nicho laboral al alcance de los hombres caboverdianos hasta mediados de 2000.

El asentamiento de la comunidad peruana se inició rozando el 2000, aprovechando los convenios bilaterales. En 1998 llegaron 24 marineros peruanos, que constituyeron el germen de una comunidad que se fue formando en los años siguientes como resultado de procesos de reagrupación familiar y de la llegada de trabajadores peruanos desde otros puertos de la cornisa cantábrica y de Galicia, donde constituyen el mayor colectivo extranjero vinculado a la pesca (Oca, 2013).

En la misma época se reactivó la corriente migratoria caboverdiana, después de un período de crisis en los años noventa. Esta migración se debió a la iniciativa privada de las familias de largo asentamiento en la zona, que consiguieron articular una ruta migratoria regular a través de Dakar, que tuvo como consecuencia la cuadruplicación del tamaño de la comunidad entre 1998 y 2011, que pasó de estar compuesta por 140 individuos a más de $550^{6}$ (Oca, 2013).

A mediados de la primera década del siglo XXI, la comunidad peruana llegó a sobrepasar a la caboverdiana, aunque solo por un breve período. El flujo peruano experimentó un crecimiento más acelerado en el período de 2004 a 2009, en que su comunidad pasó, según el Padrón, de 25 a 231 personas. En 2015 estaban censadas 124 personas peruanas y existía un equilibrio total entre los sexos en los datos de ese año, lo que da cuenta de la importancia de los procesos de reagrupación familiar y del decrecimiento del colectivo en años recientes.

A mediados de la primera década del siglo XXI, otros colectivos llegaron a la localidad para trabajar en la pesca. Destacan los marineros indonesios, llegados

6. Datos procedentes de censo (Oca, 2013). Las cifras son sensiblemente superiores a las oficiales, pues consideran a la comunidad en cuanto grupo étnico, independientemente de la nacionalidad o de la generación. Cuando en este trabajo nos referimos a datos oficiales (Padrón), somos conscientes de que el tamaño de las comunidades migrantes es siempre superior, en este caso debido fundamentalmente a las nacionalizaciones. 
a través de empresas intermediarias y sujetos a diferentes condiciones laborales que los anteriores, las cuales han sido repetidamente denunciadas por los sindicatos $^{7}$. El colectivo indonesio es actualmente el más numeroso de los dedicados a la pesca de altura, puesto que supone el 39\% de los tripulantes extranjeros en 2011 (Oca, 2013). Se trata de un grupo estrictamente masculino, ya que estos migrantes vienen con un contrato de duración determinada y retornan después a su país sin promover procesos de reagrupación.

Otro colectivo llegado en la misma época procede de Senegal, inicialmente a través de los convenios bilaterales y sirve como base para la llegada posterior de otros compatriotas desde diferentes puntos de la península a través de las redes familiares. Este colectivo, que ha crecido hasta llegar a los 58 individuos en 2015 (Padrón), presenta una elevada masculinización, que se ha ido atenuando levemente con la llegada de algunas mujeres a través de la reagrupación familiar, un modelo minoritario en los flujos senegaleses que responden a un esquema de emigración masculina individual (Vázquez, 2014). Las mujeres residentes no llegaban a representar el $14 \%$ de su comunidad en 2015 (Padrón).

Los datos indican que la inmigración en Burela ha continuado, incluso en los peores años de recesión económica. La pesca ha sido el motor de dicha continuidad. El sector de la construcción ha desempeñado un papel secundario en el mercado de trabajo inmigrante, por lo que se ha resentido en menor medida que en otras zonas de Galicia. En los dos colectivos en estudio, la migración no se ha detenido debido a la existencia de procesos de reagrupación familiar, que continuaron aportando mujeres y menores una vez se detuvo la migración laboral a través de ofertas, en torno al año 2009.

Por estudios previos, es sabido que las características de la actividad pesquera están en la base de una determinada organización de las relaciones de género en las sociedades dependientes del mar, en las que la ausencia del elemento masculino hace menos rígida la división sexual del trabajo y la propia segregación sexual. Las normas sociales de las comunidades pesqueras, incluyendo los mandatos de género, responden a necesidades prácticas y mudan conforme lo hacen estas (McGoodwin, 2001). En el caso gallego, ha sido ampliamente documentada la presencia de las mujeres en la vida exterior al ámbito doméstico desde diversos puntos de vista, como el parentesco, la sexualidad, la división sexual del trabajo, las atribuciones respecto a la gestión del agregado familiar, etc., hasta el punto de definir la sociedad costera como un «matriarcado» (Lison, 1979), idea que ha sido rebatida por múltiples estudios posteriores (Gondar, 1991; Alonso y Roseman, 2001). En la sociedad gallega existe una tradición de representación a través de las mujeres, aunque

7. El sindicato Conferencia Intersindical Galega (CIG) ha denunciado las condiciones salariales de estos marineros, que perciben retribuciones fijas muy inferiores a las de sus compañeros de tripulación. Además, las empresas intermediarias les proporcionan alojamiento y manutención descontándoselos de sus salarios, y también han sido denunciadas por las condiciones en que se prestan (Oca, 2013). 
con limitaciones, pues nunca deja de ser el hombre el que ocupa los lugares preferentes de representación en una sociedad que, como las de su entorno, es de cuño patriarcal.

\subsection{El género como elemento vertebrador de las migraciones}

Algunas tendencias teóricas recientes en el campo de las migraciones consideran el género como un principio de estructuración social caracterizado por su plasticidad y adaptación a los contextos, que supone la variabilidad de los papeles y de las relaciones de género, vistas como algo fluido y en constante dialéctica con otros elementos de la realidad (Donato et al., 2006). Esta idea se puede aplicar a las comunidades en análisis, partiendo del conocimiento de sus sociedades y de sus culturas de origen para analizar los cambios que se producen en las relaciones de género en ambos lados del proceso migratorio.

Entre las dos comunidades migrantes que cuentan con asociaciones en Burela, se pueden definir una serie de paralelismos y diferencias. Los países de origen son diametralmente opuestos en muchos aspectos, entre los que se puede señalar, en primer lugar, el pequeño tamaño del archipiélago caboverdiano, poblado por algo más de medio millón de personas, que contrasta con la gran extensión del territorio peruano, poblado por algo más de 31 millones de habitantes de diversos grupos étnicos.

La comunidad caboverdiana procede de varias pequeñas localidades de la costa oeste de la isla de Santiago $\left(991 \mathrm{~km}^{2}\right)$. Sus miembros estan vinculados por tupidas redes de parentesco y vecindad, trasladadas y reconstruidas en el espacio migratorio. En cambio, la comunidad peruana agrupa a personas llegadas de diversos hábitats (rural, urbano, montaña, costa) de un país que supera el millón de quilómetros cuadrados. Estas personas son, a menudo, desconocidas entre sí con anterioridad al proceso migratorio, y sus relaciones se han construido en la sociedad de destino. Se trata, en la mayoría de los casos, de marineros cualificados con experiencia en la pesca industrial, algo que no sucede con los colegas del archipiélago africano, procedentes de la pesca artesanal.

En ambos casos se trata de migraciones con un fuerte componente familiar, en las que los hombres son considerados el sujeto principal, pionero, mientras las mujeres ocupan un lugar subalterno, en calidad de esposas, dependientes de la decisión de sus maridos de desencadenar el proceso de reagrupamiento del hogar. Este esquema migratorio, basado en el trabajo masculino, ha llevado a la constitución de comunidades formadas mayoritariamente por familias, en las que, a pesar de existir una leve masculinización, son muy importantes los colectivos de mujeres adultas y descendientes de ambos sexos.

Las mujeres caboverdianas migraron exclusivamente como esposas durante algo más de dos décadas. Este hecho tenía como consecuencia un estatuto de residencia sin derecho a trabajo y, por tanto, dependiente del marido. La proliferación de ofertas de empleo en sectores feminizados a mediados de la primera década del siglo XXI dio pie a una pequeña corriente de mujeres migrantes no esposas (parientes de las familias ya establecidas, muchas de ellas madres 
solteras en el país de origen), lo que inauguró un flujo de migración laboral femenina que se vio paralizado con la llegada de la recesión económica.

Por su parte, la comunidad peruana está formada bien por familias, como resultado del mismo tipo de procesos de reagrupación, o bien por hombres solos, y no existen casos significativos de migración laboral femenina. Su llegada más tardía se ha dado en un momento en el que se conseguía sin dificultad permiso para trabajar legalmente, derecho al que se acogieron también las caboverdianas a partir del año 2000.

Existen no pocos paralelismos entre las mujeres peruanas y caboverdianas, más allá de los trayectos migratorios y la composición de los flujos. Entre ellos, la religión católica, mayoritaria en las sociedades de origen, cuya ideología patriarcal influye claramente en la configuración de los papeles y de las relaciones de género, en ambos casos basadas en la autoridad masculina y el papel subalterno de las mujeres. Nos parece importante señalar que en ninguno de los casos aquí traídos existe reclusión femenina, puesto que en las sociedades de origen existe una tradición de actividad productiva externa al hogar, anterior al proceso migratorio, que influye en las elevadas tasas de actividad de las mujeres migrantes en la sociedad receptora. En ambos casos, el modelo de mujer ama de casa es minoritario.

La característica común fundamental para nuestro análisis es la experiencia de estas mujeres como esposas o compañeras de marineros de pesca de altura. La profesión de los hombres determina su ausencia durante períodos más o menos largos, que oscilan entre 15 o 20 días y varios meses, lo que deja a las mujeres en una posición potencial de poder en sus hogares y de representación de sus comunidades ante la sociedad. Consideramos que este elemento común, circunstancial, es el principal factor explicativo de la existencia de un asociacionismo regido por mujeres. La ausencia de los hombres en la vida cotidiana las ha llevado a ellas a desempeñar la representación de sus colectivos, cuestión que siempre ha sido frecuente para el colectivo femenino gallego en la misma situación. Un hecho que refuerza esta idea es que las asociaciones de ambas nacionalidades, en otros puntos de Galicia y del Estado, no presentan en ningún caso esta configuración de género, siendo sus directivas mixtas o mayoritariamente, cuando no exclusivamente, masculinas.

Las vidas de estas mujeres están marcadas por la incertidumbre, tanto respecto del regreso del compañero como del salario que percibirá, en uno de los empleos con mayor riesgo. De hecho, a partir del año 2000, se han producido varios naufragios ${ }^{8}$ y accidentes laborales en los que han muerto varios marineros de ambas nacionalidades, así como de otras que también son significativas en el sector.

Conviene destacar aquí que la ausencia masculina coloca en una posición ambivalente a las mujeres de marineros, que, por el lado negativo, se enfrentan

8. Destacamos aquí el naufragio del volantero Siempre Casina, el 22 de febrero de 2005, en el que fallecieron seis tripulantes (un gallego, un caboverdiano, dos senegaleses y dos peruanos) $\mathrm{y}$ otros dos desaparecieron (un gallego y un peruano). 
en solitario a muchos hechos vitales, pero, por el positivo, tienen la oportunidad de liberarse, aunque sea temporalmente, de las obligaciones matrimoniales y domésticas (Oca, 2103, 2017).

\subsection{Asociacionismo migrante versus políticas locales: ¿Instrumentalización o integración?}

En la actualidad existen en Burela, según fuentes municipales, cinco asociaciones de inmigrantes, de las que ya se ha hablado. Todas ellas están en manos de mujeres, aunque en cuatro existen hombres asociados, teniendo vocación comunitaria, en base a familias.

Las comunidades indonesia y senegalesa no cuentan con estructuras que las representen. En el primer caso, debido a sus características de flujo estrictamente laboral de carácter temporal. El colectivo como tal se ve imposibilitado de desarrollar una vida comunitaria, pues no hay mujeres que permanezcan en tierra y los hombres siempre lo hacen de forma escalonada, de manera que no tienen ocasión de relacionarse entre ellos más que en pequeños grupos. En el segundo, pueden ser considerados factores explicativos su pequeño tamaño y reciente asentamiento. Otro factor clave que puede explicar la inexistencia de una asociación senegalesa es la escasez de mujeres que, siguiendo los ejemplos de caboverdianas y peruanas, pudiesen hacerse cargo de la organización comunitaria. En este punto se puede aventurar, a modo de hipótesis, que el sistema de relaciones de género y la segregación sexual de la sociedad de origen pueden actuar como factor inhibidor para la formación de una asociación senegalesa, en la que no sería bien vista una reproducción de los patrones de género que presentan las otras comunidades en sus organizaciones.

Las asociaciones inmigrantes han sido consideradas como estructuras de mediación entre estas comunidades y las instituciones (Cucó, 2004). Gadea y Albert (2011: 12) afirman que se constituyen "como instancias de interlocución con las instituciones públicas, para la defensa de los intereses y los derechos de los migrantes», funcionando como vehículos o herramientas para la participación sociopolítica, la cual adquiere una intensidad más visible en los ámbitos locales. Así pues, en el caso que nos ocupa, el ayuntamiento de Burela ha ido construyendo una imagen de municipio solidario y atento a las necesidades de los colectivos migrantes, con lo que la integración se presenta como una de las imágenes de marca de la localidad. En ella fue creada en 1987 la Asociación Cultural Tabanka, probablemente la más antigua de las agrupaciones de inmigrantes de Galicia.

Esta asociación nació gracias a la intervención de un líder comunitario, militante del partido de la independencia de Cabo Verde (PAICV), que trabajó en favor de la creación de asociaciones en los enclaves de la diáspora caboverdiana en la península ibérica. Durante sus dos años iniciales, la asociación visibilizó a la comunidad caboverdiana, situándola ante la sociedad como actor político, realizando diversas actividades de difusión cultural y colocando sobre la mesa problemas vinculados al área de los derechos de ciudadanía centrados 
en las situaciones de irregularidad. A partir de 1989, con la marcha del líder, la Asociación Cultural Tabanka se sumió en la inactividad, a pesar de continuar registrada y contar con una directiva que, en poco tiempo, fue integrada únicamente por mujeres (Oca, 2013).

La intervención con migrantes en la localidad no se inició desde la Administración local, sino desde una ONG de ámbito gallego con sede en A Coruña (REGAL). Dicha organización desarrolló, entre 1998 y 2000, un proyecto con la comunidad caboverdiana a través de fondos europeos de la iniciativa INTEGRA, que se conoció como Proyecto Bogavante. Consistió en una intervención basada en la proximidad y la confianza, propias del método etnográfico aplicado a la intervención social e inspirada en las ideas de integración multidimensional (Giménez, 2003; Laparra, 2003) y en la metodología del empoderamiento (Stromquist, 1995). Cuestionó la idea de «integración perfecta» mantenida por el gobierno local (Oca, 2013, 2017). Entre sus resultados se cuentan, por un lado, el acceso de migrantes y descendientes a diversos derechos civiles y sociales; por otro, la difusión de su realidad social y cultural, y, finalmente, la reactivación de la asociación comunitaria, que llevaba una década inactiva. El diagnóstico en el que se basó la intervención situó las relaciones interétnicas en Burela en el modelo de separación (Sabatier y Berry, 1996), definido por el mantenimiento de la identidad y de las prácticas culturales, con escasas relaciones positivas entre miembros de la comunidad migrante y la autóctona. Gracias a su metodología y a su enfoque, logró abrir brechas en este modelo, a pesar de su corta duración.

El municipio de Burela inició su atención específica al colectivo inmigrante en marzo de 2000, cuando, con financiamiento autonómico, se sustituyó la intervención anterior liderada por la ONG, vaciándola de todo sentido empoderador y ampliándola a toda la población migrante de la localidad, lo que coincidió con la llegada del colectivo peruano. Durante la investigación etnográfica, se ha podido constatar que la estructura del servicio municipal a la población inmigrante se ha ido centrando en un tipo de intervención burocratizada y asistencialista, encarnada en la Oficina de Atención á Poboación Inmigrante, donde fundamentalmente se apoya la resolución de trámites administrativos y se impulsan algunas actividades en colaboración con las propias asociaciones, a quienes auxilia en sus deberes administrativos y cuestiones burocráticas. Esta oficina se ha centrado desde su inicio en los colectivos caboverdiano y peruano, que han protagonizado numerosas noticias vinculadas a ella.

Volviendo a las asociaciones de Burela, la Asociación Cultural Tabanka se reactivó en 1998, en el marco de la primera intervención, lo que promovió nuevamente el carácter de actor social de la organización comunitaria a través de las mujeres que formaban su junta directiva, que emergieron como protagonistas de un proceso de empoderamiento. La asociación ha continuado activa hasta la actualidad, con altos y bajos. A partir de 2000, mediatizada por la intervención municipal, ha ido perdiendo el carácter reivindicativo que estuviera tan presente en su creación y en su posterior reactivación. 
En 2005 fue legalizada la Asociación Batuko Tabanka ${ }^{9}$, formada por las integrantes del grupo de música tradicional ${ }^{10}$, que se desgajó de la anterior asociación para concretar sus objetivos, sus actividades y sus cuentas de una forma diferenciada. Durante los años que precedieron a la creación de esta nueva organización, el grupo musical se había identificado cada vez más con la organización comunitaria, lo que en cierto modo frenaba la adhesión de socios y socias de la comunidad. Se trata de la única asociación estrictamente femenina de las cinco en estudio. Tiene unos objetivos limitados, en torno a la actividad del grupo, sin presentar una vertiente comunitaria, pues en el momento de su creación esta ya estaba ocupada por la organización «madre», con la que en cierto modo se ha seguido confundiendo, pues las mujeres integrantes del grupo musical pertenecen también a la organización comunitaria, en la que ostentan algunos cargos.

Cultura Tabanka es la asociación de todos los caboverdianos que quieren asociarse y Batuko Tabanka somos las mujeres, la hicimos nosotras, que somos las del grupo. (Entrevista a la líder de Batuko Tabanka)

Durante el proceso de rápido crecimiento de la comunidad caboverdiana, fueron apareciendo subgrupos en su seno, y, a mediados de la década de 2000, surgieron una serie de conflictos entre las migrantes de mayor o menor tiempo de residencia. En 2006 surgió un nuevo grupo de música, Batuko Rabentola, cuyas integrantes afirmaban no encontrar en la organización anterior espacio para sus actividades. Los años iniciales de convivencia entre los grupos de Batuko fueron tensos y conflictivos. La rivalidad se mantuvo a través de rumores y comentarios informales que circulaban en el interior de la comunidad. Tres años después, fue legalizada, con asesoramiento municipal, la tercera asociación caboverdiana, Asociación Cultural, Recreativa, Deportiva Rabentola, a partir del grupo musical, integrado por mujeres llegadas en la primera década del siglo XXI.

Los lazos basados en el origen y en la identidad común habían mantenido a la comunidad caboverdiana unida a lo largo de dos décadas, a pesar de existir diversos conflictos en su interior, que nunca llegaron a romper la sociabilidad preferente entre sus miembros, que reconocían a la Asociación Cultural Tabanka como organización común. Con el crecimiento de la comunidad, estos lazos se reconfiguraron en torno a nuevos ejes, como la localidad de origen o la edad. En este contexto, lo que comenzó como un conflicto entre dos generaciones migrantes, acabó por convertirse posteriormente en un conflicto

9. Batuko Tabanka es el nombre de un grupo musical nacido en 2001 como consecuencia de la intervención Bogavante, formado inicialmente por 12 mujeres adultas de la comunidad, que habían participado activamente en sus actividades. El grupo ha protagonizado desde su creación innumerables actuaciones, piezas informativas y documentales (Carnacea, 2011).

10. El batuko es un género musical interpretado mayoritariamente por mujeres, propio de la isla de Santiago, conocido dentro del panorama musical del archipiélago como el que presenta más claramente las raíces africanas. 
entre dos facciones heterogéneas de la comunidad. Dicho conflicto llevó al paso de varias de las fundadoras de Batuko Tabanka al grupo rival, de forma escalonada, al mismo tiempo que migrantes recién llegadas se iban incorporando a uno u otro colectivo, según las lealtades marcadas por las cadenas migratorias que habían facilitado su migración.

En resumen, en los últimos años las asociaciones caboverdianas funcionan como dos grupos diferenciados y rivales. Ambas cuentan con un grupo musical como forma de expresión más visible. Se ha ido configurando un nuevo escenario en que la representación de la comunidad, mucho más numerosa y compleja, se encuentra escindida.

Por otro lado, la comunidad peruana se organizó inicialmente de manera informal. Durante el periodo 2005-2006 se llegó a fundar, aunque no a legalizar, con apoyo municipal, una asociación peruana, que no fue adelante por conflictos entre diferentes subgrupos, encabezados por dos mujeres. Es decir, de nuevo conflictos personales entre líderes que entorpecían la organización comunitaria en presencia de la Administración. Datan de esta época numerosas noticias vinculadas a la oficina municipal sobre organización de fiestas típicas peruanas. La primera organización comunitaria andina no nació oficialmente hasta 2011, algo más de una década después de la llegada de los pioneros. Se trata de la Asociación de Peruanos de Burela (ASPEBU ${ }^{11}$ ), que se dedica a organizar actividades lúdicas conmemorativas de fechas especiales para la sociedad peruana:

Nosotros celebramos el día de la Madre, el del Padre, el 20 de Julio, el Día de la Independencia y ahora la Primavera. (Entrevista a la presidenta de ASPEBU)

Un año después fue legalizada la Asociación Sociocultural Raíces Peruanas de Burela ${ }^{12}$ (2012) a partir de una escisión de la anterior, y fue patente el conflicto que llevó a esta ruptura, con acusaciones entre sus dirigentes. La vicepresidenta de ASPEBU abandonó la organización con un grupo de socias y se convirtió en la presidenta de la nueva asociación.

Siempre hubo diferencias de personas y, ante ello, se decidió, con un grupo de peruanos, salir de ahí y formar otra asociación, porque no era la única institución que podía representarnos, ¿̨no? Que también podíamos nosotros, este, formar otra asociación y trabajar. Entonces es lo que hemos estado haciendo hasta ahora. (Entrevista a la presidenta de Raíces Peruanas)

Según su presidenta, Raíces Peruanas persigue fines de bienestar y ayuda a los socios, por un lado, y de intercambio cultural, por otro, a través de música, danza y gastronomía. Sus celebraciones y fiestas coinciden con las efemérides señaladas por la asociación ASPEBU.

11. Según su presidenta, contaba en 2015 con 63 personas asociadas, con casi 50 menores, que pagaban una cuota mensual de 3 euros por persona.

12. Según su presidenta, contaba en 2015 con 14 familias asociadas que pagaban una cuota mensual de 5 euros por persona. 
En resumen, se constata que las cuatro asociaciones migrantes más recientes de Burela han nacido vinculadas a la oficina municipal, lo que responde en cierto modo a sus propuestas. La intervención institucional se limitó al asesoramiento y al acompañamiento de los procesos burocráticos de legalización, sin realizar una intervención «de fondo" que trabajase por la superación de los conflictos y que permitiese realizar la interacción positiva de las organizaciones rivales. Como resultado de estos procesos, cada una de estas comunidades migrantes funciona, a efectos asociativos, como dos grupos escindidos.

\subsection{Trayectorias asociativas: Entre la instrumentalización y la rivalidad.}

Como resultado del proceso que venimos analizando, la actividad asociativa ha estado en gran medida mediatizada por un poder local que concentra sus acciones de apoyo en dos líneas: una, de corte asistencialista, y otra, que podemos denominar folclórica o culturalista.

La intervención asistencialista se limita al apoyo en la tramitación de expedientes administrativos. En este ámbito, ligado a lo social, ha desaparecido por completo el discurso de los derechos, a pesar de la existencia de múltiples problemas a lo largo de estos años (irregularidad sobrevenida, trabas al empadronamiento de irregulares, problemas en el acceso a la sanidad, fracaso escolar, etc.). En esta vertiente, la intervención municipal no se ha dirigido a las comunidades, sino a los individuos o a las familias, abandonando la perspectiva colectiva, sin considerar a las asociaciones en su función de representación conjunta ante las instituciones. Se evita, de este modo, un eventual surgimiento de demandas grupales en el ámbito de los derechos (Oca, 2013). En esta lógica, la división y el conflicto entre las asociaciones no hace sino favorecer el statu quo, impidiendo en cierto modo que las organizaciones migrantes cumplan una de sus funciones principales, la de mediación ante las instituciones y de traslado de las necesidades y demandas de los colectivos.

Junto al enfoque individualizado, la intervención municipal puede ser definida como «desde fuera», en contraposición al método etnográfico que sustentó la acción y la investigación inicial del Proyecto Bogavante. Con el paso del tiempo, a través de los datos recogidos y del análisis realizado, se constata que la propia actuación municipal, lejos de servir como cauce para el empoderamiento de los colectivos migrantes, ha aprovechado las situaciones de conflicto y de ruptura intracomunitarias en su beneficio, trabando, de esta manera sutil, el surgimiento de demandas colectivas.

En cuanto a la línea culturalista, se aprovecha la voluntad de las comunidades de recrear y difundir sus prácticas culturales, utilizándolas en la construcción de la imagen de «integración perfecta». Tanto las asociaciones peruanas como las caboverdianas cuentan con grupos de música y de baile que suelen realizar actividades de difusión cultural. En las actividades promovidas por el municipio, estos grupos funcionan como encarnación de la integración. Son el espejo o, más bien, el elemento de legitimación que el Ayuntamiento utiliza para constatar la existencia de políticas de integración exitosas. 
No se ha considerado a las asociaciones migrantes como actores políticos, pero sí como soportes de la comunidad étnica, en el sentido identitario. La intervención se ha aprovechado aquí de la función de «microclima cultural» (Albert, 2004: 12) propia de las asociaciones migrantes, promoviendo puntualmente su exposición pública, sin conectar los elementos culturales con los sociales. Como resultado, existe una profunda brecha entre lo que se muestra y la realidad social comunitaria. Ha desaparecido el enfoque intercultural inicial, desaprovechando la oportunidad de utilizar las prácticas culturales de las sociedades de origen como herramientas hacia una integración multidimensional, más allá de su exposición repetitiva.

Esta consideración ha dado lugar a una situación que satisface las necesidades de identificación de las personas migrantes, pero no su integración. Como resultado, se produce una paradoja (Merino, 2005), pues actividades que supuestamente responden a objetivos de integración acaban por tener como efecto un refuerzo del modelo de separación (Sabatier y Berry, 1996). Estos eventos difícilmente consiguen llegar a generar diálogo e intercambios con la población autóctona, que los ve como "privados».

En este sentido, una de las entrevistadas peruanas ha expresado sus quejas por el escaso interés, no solo institucional, sino también de la población en general, en participar en este tipo de actividades, en comparación con otras no vinculadas a la comunidad migrante.

Burela, se hizo, participaron inmigrantes, muy bien. Participó Cabo Verde y participó Perú, pero ellos nos piden que nosotros nos integremos, integrar, formar, integración, comentar, muy bien... Y entonces dicen que nosotros nos integremos y eso se trata para que lo vean todos, tanto españoles como caboverdianos, todos. Porque nos llaman para que nosotros nos integremos, nosotros nos queremos integrar, pero nos echan para atrás. En ese sentido, para qué nos esmeramos tanto, también estas niñas, se esmeraron en ensayar todo, también para vernos a nosotros mismos... Y ese día por qué, solo porque dicen inmigrante y ya no van. Y cuando fue del día siguiente, que fue del karaoke y lleno, que no cabía ni una aguja. (Entrevista a la presidenta de ASPEBU)

Los esfuerzos de las asociaciones se han centrado en la celebración de actividades festivas y existe una especie de competición entre ellas, lo que ha vaciado las estructuras comunitarias de otras funciones. Por lo tanto, su valor comunitario se resume en funcionar como un espacio de sociabilidad y de expresión de la identidad, a través de la reproducción y de la recreación de ciertas prácticas culturales que, a lo largo del año, en la mayoría de las ocasiones, se realizan sin la participación institucional, más centrada en promoverlas y apoyarlas en determinadas efemérides locales.

La relación con las asociaciones ha evitado de esta forma el ámbito social de la intervención, centrándose en el plano cultural. Como interlocutoras principales ante la Administración y los medios de comunicación se ha utilizado a las representantes de asociaciones, a quienes es habitual ver en la oficina municipal, especialmente para exponer demandas de ayuda para la realización 
de actividades. Esta oficina centraliza la información sobre subvenciones. Es la encargada de difundirlas y de apoyar a las asociaciones en una eventual petición de fondos para desarrollar sus actividades. Nos consta que las asociaciones caboverdianas han sido las mayores beneficiarias de financiación institucional, mientras que las líderes peruanas han expresado sus quejas por no haber logrado acceder a este tipo de ayudas.

Por último, queremos destacar que ambos colectivos están presentes en las listas a las elecciones municipales. Siempre han sido mujeres las candidatas de origen extranjero, desde su aparición en las listas del BNG y del CDS en 2003, que se ha ido ampliando posteriormente hasta cubrir la totalidad del espectro político en la última convocatoria.

Existe una tercera función asociativa, potencialmente más ajena a las instituciones que las anteriores, que se centra en la solidaridad entre sus miembros (Rex, 1994; Albert, 2004). La función de solidaridad es, según alguna de las representantes comunitarias, la única en la que las asociaciones, rivales, dejan a un lado sus conflictos para centrarse en la ayuda común:

Si tenemos que ayudar pasamos de esto y ayudamos igual, ya no hay Rabentola y no hay Tabanka [...] ya pasamos de la asociación y vamos a los caboverdianos, porque hay cosas que no se pueden mezclar con otras. (Entrevista a la presidenta de Rabentola)

Las situaciones desencadenantes de esta solidaridad grupal, que transcienden las fronteras asociativas, los conflictos y las desconfianzas, se concretan especialmente con motivo de ritos asociados a la muerte, que son reproducidos en el contexto migratorio. Los naufragios se han convertido en ocasiones privilegiadas para la expresión de la solidaridad interétnica entre latinos y africanos, con base en la dura y peligrosa experiencia laboral común y en las prácticas religiosas, también comunes.

Siempre ha existido una participación activa en los asuntos religiosos por parte de ambas comunidades, que en los últimos años se ha tornado más evidente y visible. Durante décadas la iglesia fue el lugar de celebración por excelencia de los rituales festivos de la comunidad caboverdiana. En la actualidad, peruanos y otros colectivos latinos también participan en estas celebraciones. Una de las asociaciones peruanas ha constituido la Hermandad del Señor de los Milagros, que participa activamente en la Semana Santa de la localidad. La asociación Rabentola celebra cada año, desde 2010, la festividad de Nossa Senhora dos Navegantes, que incluyó la entrega de una imagen religiosa a la parroquia. Esta celebra todos los años misas interculturales en las que se produce una comunión entre peruanas y caboverdianas marcadas con sus elementos étnicos.

Más allá de las estructuras asociativas, la solidaridad, totalmente alejada de ámbitos institucionales, es practicada de forma cotidiana con el intercambio de bienes y cuidados, en especial los que posibilitan la conciliación laboral y familiar de las mujeres con trabajo extradoméstico. En ambas comunidades 
existen redes de solidaridad interdoméstica protagonizada por mujeres, que no solo vertebran las asociaciones, sino también las propias comunidades.

\section{Conclusiones y futuras líneas de investigación}

Una vez analizados los datos sobre la presencia y la representatividad de las mujeres en las asociaciones migrantes actualmente activas en Galicia y de estudiar en profundidad un caso significativo, podríamos afirmar que la existencia de asociaciones regidas por mujeres no parece corresponderse con una consciencia feminista o con una sociedad normalizada en cuestiones de igualdad de género, sino que responde a otros factores. En algunos casos, responde a procesos de fuga respecto al machismo y a la segregación sexual, vinculados a la religión o a las prácticas patriarcales que arrastran de las sociedades de origen. En otros casos, como el de Burela, debido a circunstancias coyunturales que han colocado a las mujeres como representantes de sus colectivos. En otras ocasiones, como sucede con las asociaciones de origen latino, han sido las propias capacidades de las mujeres y su sensibilidad hacia cuestiones ligadas a roles de género, en concreto, al ejercicio de la maternidad, derivada de su preocupación por que sus hijos se integren en las sociedades de acogida de una manera satisfactoria, para poder experimentar situaciones de movilidad social ascendente, que repercuta en una mejora de las condiciones de vida, no solo familiares, sino también de la propia comunidad.

Las entrevistas realizadas permiten constatar la escasa incidencia de las llamadas políticas de integración más allá del ámbito municipal, especialmente en las ciudades y en las localidades que cuentan con algún servicio especializado de atención a las poblaciones migrantes. Las administraciones no saben llegar a conectar con la población migrante, ni mucho menos consiguen responder a sus demandas, puesto que orientan su trabajo hacia aspectos que tienen que ver más con lo folclórico y con lo identitario que con actividades que fomenten un verdadero diálogo e intercambio entre iguales, lo que debería incluir el trabajo con la población autóctona, y no solo con las comunidades migrantes.

En cuanto a la existencia de medidas favorables a las mujeres migrantes, es notable su ausencia en las políticas autonómicas. No parece tampoco que, salvo raras excepciones, las intervenciones locales estén orientando sus acciones hacia la igualdad de género, en el sentido de apoyar y promover la presencia y el liderazgo femeninos en las organizaciones migrantes. Los datos recogidos muestran que la llegada de las mujeres a los cargos representativos de sus comunidades obedece a factores circunstanciales, cuando no a la propia decisión de los grupos de mujeres (en uno de los casos, apoyadas por la Administración local) de contar con un espacio propio de trabajo, abandonando la posición subalterna que les otorgan sus comunidades.

En cuanto al liderazgo femenino, más allá de las cuestiones apuntadas anteriormente, conviene destacar la importancia de la situación y de las capacidades personales que presentan las mujeres líderes. En el caso de las latinas, se evidencia la relevancia de las experiencias previas en sus países de origen. No 
así en las africanas, ya que el tejido asociativo, por lo general, suele ser mucho más débil que el contexto latinoamericano, que cuenta con una amplia tradición de movimientos sociales y organizaciones con amplia presencia femenina y con liderazgos muy fuertes, lo que facilita que asuman un papel relevante en las sociedades de acogida.

Con este trabajo se demuestra que la igualdad en las relaciones de género no es una cuestión que se deba abordar exclusivamente desde una perspectiva cuantitativa. El caso de estudio, basado en datos etnográficos, arroja una serie de conclusiones que contrarían la idea inicial que podrían haber sugerido los datos cuantitativos, de una sociedad local igualitaria en la que las mujeres migrantes ocupan claramente espacios de representatividad y toma de decisiones.

En primer lugar, se ha constatado que la excepcionalidad del caso de Burela y sus cinco asociaciones migrantes encabezadas por mujeres viene dada por un sistema social dependiente de la actividad pesquera, que las ha colocado a ellas al frente de sus colectivos de forma circunstancial, debido a la ausencia masculina, cuestión que se repite en el resto de la sociedad local.

En segundo lugar, se ha producido una instrumentalización de las organizaciones migrantes por parte del poder local, que se ha basado en la exposición y en la repetición de determinadas prácticas culturales, utilizándolas como elemento legitimador de una imagen de integración, entendida de una forma culturalista, lejos de contenidos emancipadores. El protagonismo de las mujeres en el tejido asociativo migrante suma argumentos en la legitimación de esta imagen de integración y modernidad por parte de los poderes locales, que al mismo tiempo instrumentalizan a las mujeres migrantes para la obtención de apoyos ligados a las políticas de igualdad y no solo a las políticas de integración. A pesar de los cambios políticos que se han ido produciendo a lo largo de los años, la intervención municipal no ha conseguido hasta ahora ${ }^{13}$ redefinirse hacia un modelo más participativo, en el que las asociaciones puedan efectivamente ejercer el papel de mediadoras, más allá de servir como elemento propagandístico (Oca, 2013, 2017) de los sucesivos gobiernos municipales.

La proliferación de entidades asociativas por parte de los colectivos migrantes en Burela responde a un aprovechamiento de los conflictos y de las rivalidades internas por parte de la intervención municipal, que se ha mostrado incapaz de cumplir una función mediadora, puesto que ha apoyado la creación de nuevas asociaciones, lo que ha incrementado las rivalidades en el seno de los colectivos. Las asociaciones han caído, así, en una paradoja, cerrándose en un espacio que limita sociabilidades, que reproduce prácticas identitarias que dificultan la integración multidimensional y la solución de problemas vinculados a la realidad social de explotación y discriminación que experimentan las personas migrantes (Merino, 2005).

13. En las elecciones municipales de 2015, fue elegida, en la lista del PSOE, una joven descendiente de caboverdianos que ocupa la Concejalía de Inmigración y Cooperación. En el momento del trabajo de campo se encontraba en los meses iniciales de mandato. 
En este sentido, las asociaciones se convierten en escenario de estrategias orientadas a la obtención de beneficios de las instituciones públicas, a la vez que en espacio de luchas por la capacidad de representación, con el objetivo de captar fondos o gestionar recursos (Garreta, 1998). Más allá del espacio local, estas luchas se repiten en la articulación de diversas asociaciones de un mismo origen en el espacio autonómico, como es el caso de las peruanas y de las senegalesas.

En definitiva, la participación política de las mujeres inmigrantes a través de las asociaciones en Galicia está mediatizada por diversos factores, entre los que se pueden destacar en primer lugar las ideologías y las prácticas de género de sus sociedades de origen, que las empujan a la actividad asociativa, bien a partir de su negación como sujetos políticos, bien a partir de sus propias capacidades de liderazgo, ya desarrolladas con anterioridad en los países de origen. En segundo lugar, las condiciones de las sociedades de destino mediatizan asimismo esta participación, sobre todo en lo relacionado con la división sexual del trabajo de los colectivos migrantes. En este sentido, las propias condiciones de las mujeres autóctonas pueden contribuir a reforzar o a limitar el papel y el peso de las migrantes en el tejido asociativo. Finalmente, destaca el factor de las políticas públicas, que, a juzgar por los datos obtenidos, lejos de promover el empoderamiento de las dirigentes asociativas y sus comunidades, parecen responder a una agenda oculta que gravita fundamentalmente sobre la imagen de las administraciones locales.

El análisis muestra, por último, la necesidad de ir más allá de las cifras en el estudio de la participación femenina en el asociacionismo migrante, pues cada caso esconde una serie de matices y de articulaciones complejas entre factores de los tres tipos anteriores, únicamente perceptibles a través del uso de metodologías cualitativas que permitan aprehenderlos en su totalidad.

\section{Referencias bibliográficas}

Albert, María (2004). La eclosión asociativa en el tránsito hacia una nueva era: Un estudio del Tercer Sector en el ámbito comarcal de l'Horta Sud (Valencia). Valencia: Servei de Publicacions de la Universitat de València.

Alonso, Enrique y Roseman, Sharon (coord.) (2011). Antropoloxía das mulleres galegas: As outras olladas. Santiago de Compostela: Sotelo Blanco Edicións.

ApARICIO, Rosa y TORNOS, Andrés (2010). Las asociaciones de inmigrantes en España: Una visión de conjunto. Madrid: Observatorio Permanente de la Inmigración (OPI).

CARDESÍn, José María (1992). Tierra, trabajo y reproducción social en una aldea gallega (ss. XVIII $y X X$ ): Muerte de unos, vidas de otro. Madrid: SGT del MAPA.

CARnACEA, M. ángeles (2011). «Batuko Tabanka: Música entre el mar y la tierra. Mujeres cabo-verdianas de Burela». En: CARNACEA, M.a Ángeles y LoZANO, Ana (coords.). Arte, intervención y acción social: La creatividad transformadora. Madrid: Grupo 5, 3-26.

CucÓ, Josefa (2004). Antropología urbana. Barcelona: Ariel.

Donato, Katharine; GabaCCIA, Donna; Holdaway, Jennifer; Manalansan, Martin y Pessar, Patricia R. (2006). «A Glass Half Full?: Gender in Migration Studies». International Migration Review, XL(1), 3-26. $<$ https://doi.org/10.1111\%2Fj.1747-7379.2006.00001.x> 
GadeA, M. ${ }^{a}$ Elena y Albert, María (2011). «Asociacionismo inmigrante y renegociación de las identificaciones culturales». Política y Sociedad, 48(1), 9-25.

GARRETA, Jordi (1998). «Minories ètniques, associacionisme i integració sociocultural». Papers de Sociología, 56, 197-230. <http://dx.doi.org/10.5565/rev/papers/v56n0.1951>

GARRETA, Jordi y LLEVOT, Nuria (2013). «Las asociaciones de inmigrantes africanos: Organización, proyección y actuaciones». Revista Internacional de Sociología, 71(1), $15-38$. <https://doi.org/10.3989/ris.2012.09.01>

GIMÉNEZ, Carlos (2003). ¿Qué es la inmigración?, ¿problema u oportunidad?, ¿cómo lograr la integración de los inmigrantes?, ¿multiculturalismo o interculturalidad? Barcelona: RBA.

GONDAR, Marcial (1991). Mulleres de Mortos: Cara unha Antropoloxía da muller galega. Vigo: Xerais.

Gregorio, Carmen (2012). «Tensiones conceptuales en la relación entre género y migraciones: Reflexiones desde la etnografía y la crítica feminista». Revista de Sociología, 97(3), 569-590. <https://doi.org/10.5565/rev/papers/v97n3.423>

LAPARRA, Miguel (2003). Extranjeros en el purgatorio: Integración social de los inmigrantes en el espacio local. Barcelona: Bellaterra.

Lisón, Carmelo (1979). Antropología Cultural de Galicia. Madrid: Akal.

MARTíneZ BujÁN, Raquel (2008). "Envejecimiento, mercado laboral e inmigración». Revista del Instituto de Estudios Económicos, 2-3, 65-85.

Martínez de LizARrondo, Antidio (2008) «Los procesos de gestión y evaluación de las políticas autonómicas de integración de inmigrantes». Revista en Gestión y Análisis de Políticas Públicas (GAAP), 2. <https://doi.org/10.24965/gapp.v0i2.423>

- (2009) «La integración de inmigrantes en España: El modelo patchwork». Revista de Migraciones, 26, 115-146.

McGoodwING, James R. (2001). Understanding the cultures of fishing communities: A key to fisheries management and food security. Roma: FAO.

MEDINA, Claudia (2009). Los ausentes están siempre presentes: Una aproximación interpretativa de la experiencia materno-filial transnacional entre España y Colombia. Madrid: Universidad Complutense. Departamento de Sociología I (Cambio Social). Tesis doctoral. Recuperado de <http://eprints.ucm.es/12191/1/T32120. pdf>.

MERINO, M. a Asunción (2005). "Asociacionismo inmigrante y modos de incorporación a la sociedad receptora: El caso peruano». Anuario Americanista Europeo, 3, 2221 3872.

Molina, Fidel; SAmper, Luís y Mayoral, Dolors (2013). «Liderazgo femenino: Un análisis de las diferencias de género en la formación y desarrollo de asociaciones de inmigrantes africanos». RIS, 71 (extra 1), 141-166. $<$ https://doi.org/10.3989/ris.2012.09.24>

OCA, L. (2013). Caboverdianas en Burela (1978/2008): Migración, relacións de xénero e intervención social. Departamento de Filosofía e Antropoloxía Social. Universidade de Santiago de Compostela. Tesis doctoral. Recuperado de $<$ https://dspace.usc. es/handle/10347/9804>.

- (2017). "Cape Verdeans in Burela: Women's Empowerment Through Gendered Cultural Practices». En: DePalma, Renée y Pérez-Caramés, Antía (eds.). Gali- 
cian Migrations: A Case Study of Emerging Super-diversity. Nueva York: Springer, 193-206. <https://doi.org/10.1007/978-3-319-66305-0>

Putman, Robert (1993). Making Democracy Work. Princeton: Princeton University Press.

REX, Jane (1994). «Ethnic mobilization in Britain». Revue Européenne des Migrations Internationales, 10(1), 7-18.

SABATIER, Colette y BerRY, John W. (1996). «Integración y aculturación». En: BouRHIS, Richard Y. y LEYENS, Jacques-Philippe. Estereotipos, discriminación y relaciones entre grupos. Aravaca: McGraw Hill, 217-239.

SOUTELO, Raúl (2011). «La memoria social de la emigración: Recuperación, análisis temático y usos didácticos de las cartas y las fotos de familias gallegas con emigrantes». En: Blanco RodrígueZ, Juan Andrés (ed.). La emigración castellana y leonesa en el marco de las migraciones españolas. Zamora: UNED.

STROMQUIST, Nelly (1995). «The Theoretical and Practical Bases for Empowerment». En: Medel-AÑonuevo, Carolyn (ed.). Women, Education and Empowerment: Pathways towards Autonomy. Hamburgo: UNESCO, 13-22.

VÁzQUEZ, Iría (2014). La inmigración senegalesa en Galicia: Remesas y cuidados en familias transnacionales. Departamento de Socioloxía e Ciencia Política e da Administración Universidade de A Coruña. Tesis doctoral. Recuperado de <http:// ruc.udc.es/dspace/bitstream/handle/2183/12341/VazquezSilva_Iria_TD_2014. pdf? sequence $=2 \&$ isAllowed $=y>$. 